\title{
New Approaches to Estimation of White Matter Connectivity in Diffusion Tensor MRI: Elliptic PDEs and Geodesics in a Tensor-Warped Space
}

\author{
Lauren O'Donnell ${ }^{1}$, Steven Haker ${ }^{2}$, and Carl-Fredrik Westin ${ }^{1,2}$ \\ 1 MIT AI Laboratory, Cambridge MA 02139, USA \\ odonnell@ai.mit.edu \\ 2 Laboratory of Mathematics in Imaging, Brigham and Women's Hospital, Harvard \\ Medical School, Boston MA, USA \\ $\{$ haker, westin\}@bwh. harvard.edu
}

\begin{abstract}
We investigate new approaches to quantifying the white matter connectivity in the brain using Diffusion Tensor Magnetic Resonance Imaging data. Our first approach finds a steady-state concentration/heat distribution using the three-dimensional tensor field as diffusion/conductivity tensors. Our second approach casts the problem in a Riemannian framework, deriving from each tensor a local warping of space, and finding geodesic paths in the space. Both approaches use the information from the whole tensor, and can provide numerical measures of connectivity.
\end{abstract}

\section{Background}

Diffusion Tensor Magnetic Resonance Imaging (DT-MRI) measures the selfdiffusion of water in biological tissue. The utility of this method stems from the fact that tissue structure locally affects the Brownian motion of water molecules. Consequently, a coherent organization of tissue (over scales comparable to that of a voxel) will be reflected in the DT-MRI diffusion measurements.

Neural fiber tracts contain parallel axons whose membranes restrict diffusion, so the self-diffusion of water is most probable along the tracts. Thus in DT-MRI imagery of the brain, the local structure of the diffusion tensor can be treated as an approximation to the local neural fiber structure. The diffusion tensor is a low-pass, Gaussian approximation to the actual microscopic structure of the neuroanatomy, but it provides a fast and non-invasive anatomical measurement.

In DT-MRI, the diffusion tensor field is calculated from a set of diffusionweighted images by solving the Stejskal-Tanner equation (eq. 11). This equation describes how the signal intensity at each voxel decreases in the presence of diffusion:

$$
S_{k}=S_{0} \mathrm{e}^{-b \hat{g}_{k}^{T} D \hat{g}_{k}} .
$$

Here $S_{0}$ is the non-diffusion-weighted image intensity at the voxel and $S_{k}$ is the intensity measured after the application of the $k$ th diffusion-sensitizing

(C) Springer-Verlag Berlin Heidelberg 2002 
gradient. $\hat{g}_{k}$ is a unit vector representing the direction of this diffusion-sensitizing gradient, and $D$ is the diffusion tensor, so the product $\hat{g}_{k}^{T} D \hat{g}_{k}$ represents the diffusivity in direction $\hat{g}_{k}$. In addition, $b$ is LeBihan's factor describing the pulse sequence, gradient strength, and physical constants [1].

There is a physical interpretation of the diffusion tensor, $D$, which is closely tied to the standard ellipsoid tensor visualization scheme. The eigensystem of the diffusion tensor describes an ellipsoidal isoprobability surface, where the axes of the ellipsoid have lengths given by the square root of the tensor's eigenvalues. A proton which is initially located at the origin of the voxel has equal probability of diffusing to all points on the ellipsoid.

Initial work on DT-MRI connectivity focused on tractography [821], or the interpolation of paths through the principal eigenvector field. An extension of this method evolved a surface through the field using a discretized fast marching method, where the speed function was dependent on the principal eigenvector field [7]. Another approach iteratively simulated diffusion in a 2D tensor volume, and quantified connection strengths based on a probabilistic interpretation of the arrival time of the diffusion front [3]. The tractography approach has also been extended to much higher angular resolution diffusion data, and connectivity has been estimated using the most probable path between points [10].

Our first approach finds a steady-state concentration/heat distribution using the three-dimensional tensor field as diffusion/conductivity tensors. The steadystate flow along any path reflects connectivity. Our second approach casts the problem in a Riemannian framework, deriving from each tensor a local warping of space, and finding geodesic paths in the space. In this method, path lengths are related to connectivity. Both approaches use the information from the whole tensor, and can provide numerical measures of connectivity.

\section{Diffusion Theory}

Fick's first law relates a concentration difference to a flux (a flow across a unit area). It states that the flux, $j$, in any direction is proportional to the concentration gradient, $\nabla u$, in the opposite direction. The proportionality constant $d$ is the diffusivity in the direction of interest.

$$
j=-d \nabla u .
$$

For an anisotropic material, the flow field does not follow the concentration gradient directly, since the material properties also affect diffusion. Consequently, the diffusion tensor, $D$, is introduced to model the material locally.

$$
j=-D \nabla u .
$$

The standard model of diffusion says that over time, the concentration of the solute will change as the divergence of the flux:

$$
u_{t}=\nabla \cdot(D \nabla u) .
$$


This is due to conservation of mass. Intuitively it means that, for example, fluid flow outward from a point (divergence) should decrease the concentration at that point while increasing the concentration at neighboring points. In the steady state, the concentration does not change; consequently the steady-state flux vector field is divergence-free.

\section{PDE-Based Connectivity}

Previous work has employed an iterative technique to create time-of-arrival maps of a heat diffusion front [3]. Instead, we solve directly for the steady state concentration, $u$, which can also be thought of as a heat distribution in the tensor field:

$$
\nabla \cdot(D \nabla u)=0 .
$$

We use this information to create the flux vector field, $j=-D \nabla u$, which describes the steady-state heat flow in the tensor volume (eq. 3). Paths in this divergence-free vector field can be compared using a connection strength metric that approximates the total flow along the path:

$$
\int_{P}\left|j^{T} t\right| d s
$$

where $j$ is the flux along the path, and $t$ is the unit tangent to the path. Normalization for the length of the path may also be included in the metric. To obtain an overall connection strength measure between two points, the value of the maximum flow path can be taken.

Of great interest in this method are the boundary conditions, or the locations of sources and sinks in the tensor field. One possibility is to set a region or regions of interest as the source, and simulate a sink at infinity. Another useful possibility is to choose one region of interest as the source, and another as the sink. In the experiments discussed in this paper, we have simulated a sink at one point of interest, and a source at another, in order to estimate the flow between the regions.

\subsection{Experiments}

DT-MRI Data Acquisition. DT-MRI scans of normal subjects were acquired using Line Scan Diffusion Imaging [6] on a 1.5 Tesla GE Echospeed system. The following scan parameters were used: rectangular $22 \mathrm{~cm}$ FOV (256x128 image matrix, $0.86 \mathrm{~mm}$ by $1.72 \mathrm{~mm}$ in-plane pixel size); slice thickness $=4 \mathrm{~mm}$; interslice distance $=1 \mathrm{~mm}$; receiver bandwidth $=+/-6 \mathrm{kHz}$; $\mathrm{TE}=70 \mathrm{~ms}$; $\mathrm{TR}=$ $80 \mathrm{~ms}$ (effective $\mathrm{TR}=2500 \mathrm{~ms}$ ); scan time $=60$ seconds/section. 20 axial slices were acquired, covering the entire brain. This protocol provides diffusion data in 6 gradient directions as well as the corresponding T2-weighted image. All gradients and T2-weighted images are acquired simultaneously, and thus do not need any rigid registration prior to the tensor reconstruction process. Tensors are reconstructed as described in [12] and eigenvalues are computed. 
Tensor Preprocessing. We are interested in measuring connectivity in the white matter, and consequently, to de-emphasize other regions, we multiply the tensors by a soft mask. This is necessary to decrease the effect of the ventricles, where neural fiber tracts are nonexistent but water diffusion is relatively unrestricted and has large magnitude. We calculate the weights in the mask as the linear shape measure at each voxel, which lies in the range of zero to one 1112

$$
c_{l}=\frac{\lambda_{1}-\lambda_{2}}{\lambda_{1}} .
$$

In addition, we remove negative eigenvalues to ensure that each tensor is a positive definite matrix. We set a small positive lower bound for the eigenvalues to guarantee that the tensors are invertible, which is necessary when utilizing them as local metric descriptors as described below. Setting the negative eigenvalues to zero would give the closest positive semi-definite tensor in the least-squares sense, but would not ensure invertibility.

Concentration/Heat Flow between Regions. In this experiment we solve for the steady-state concentration/heat distribution in the tensor field, with boundary conditions of one source and one sink. The maximal flow is found as expected along the strong anatomical path between the source and sink, the corpus callosum. Figure 1 displays the steady-state concentration and flow.
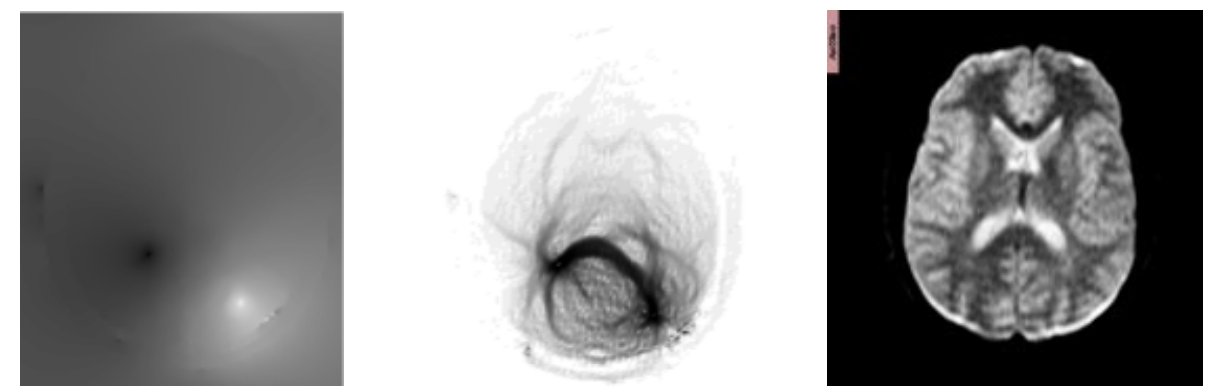

Fig. 1. Results of solving equation $[5$ for the steady-state heat distribution. The temperature (left) and the steady-state flow magnitude (center) demonstrate the flow from the source to the sink. In the temperature image the source is bright and the sink is dark; in the center flow image, dark means high flow magnitude. The grayscale image on the right, a non-diffusion-weighted image, shows the corresponding anatomy.

\section{A Riemannian Metric Space}

A natural interpretation of the "degree of connectivity" between two points is the distance between the points in some metric space. For our purposes, the distance between two anatomical locations should depend on the diffusion tensor field. 
The diffusion operator (eq. 4) can naturally be associated with a Riemannian metric tensor $G$ via the relation $G=D^{-1}$, allowing us to compute geometric quantities such as geodesic paths and distances between points in the brain. The relation between the diffusion and metric tensors is intuitive: large eigenvalues in the original DT-MRI tensor become small when the tensor is inverted to create the metric tensor. Consequently, large eigenvalues in the original tensor imply short metric distances along the direction of the corresponding eigenvector.

We will limit ourselves here to a brief discussion of the theory; see 4 for a more rigorous and thorough treatment of the connection between diffusion and Riemannian geometry. The Laplacian for a scalar function $u$ on a manifold can in tensor notation be written as:

$$
\nabla_{G}^{2} u=\left(G^{k l} u_{; k}\right)_{; l}=G^{k l} \frac{\partial^{2} u}{\partial x^{k} \partial x^{l}}-\Gamma^{l} \frac{\partial u}{\partial x^{l}}
$$

where $G_{k l}$ is the metric tensor, and $\Gamma$ is the Christoffel symbol that represents the derivatives of the basis vectors and the metric,

$$
\Gamma^{i}=\frac{1}{2} G^{k l} G^{i j}\left(\frac{\partial G_{j k}}{\partial x^{l}}+\frac{\partial G_{j l}}{\partial x^{k}}-\frac{\partial G_{k l}}{\partial x^{j}}\right) .
$$

It can be noted that the Christoffel symbol is zero when the basis vectors and the metric are spatially invariant, as in the case of $\mathcal{R}^{n}$. Inserting the expression for the Christoffel symbol from equation 8 gives

$$
\nabla_{G}^{2} u=\frac{1}{|G|^{\frac{1}{2}}} \frac{\partial}{\partial x^{k}}\left(|G|^{\frac{1}{2}} G^{k l} \frac{\partial u}{\partial x^{l}}\right),
$$

which is known as the Laplace-Beltrami operator, the generalization of the Laplacian to manifolds. In matrix notation the Laplace-Beltrami operator can be written as

$$
\nabla_{G}^{2} u=|G|^{-\frac{1}{2}} \nabla \cdot\left(|G|^{\frac{1}{2}} G^{-1} \nabla u\right) .
$$

It is straightforward to check from these definitions that we have the following relation between the diffusion operator in (eq. 4) and a diffusion operator in the Riemannian space characterized by $G$ :

$$
\nabla \cdot(D \nabla u)=\nabla_{G}^{2} u-\frac{1}{2}\left\langle\nabla \log |G|, \nabla_{G} u\right\rangle
$$

where the second order term on the right hand side represents simple Laplacian smoothing in the tensor-warped space, i.e. isotropic diffusion associated with the heat equation.

\subsection{Measuring Distances in the Tensor-Warped Space}

Once we have the metric tensor $G$, we are able to apply results from Riemannian geometry to describe geometric objects such as geodesic paths and distances 
between points in the brain. Unlike tractographic methods based on following the flow of principal eigenvectors of $D$, these geodesic paths are well-defined even in regions where the tensor diffusion is isotropic.

We have approached the measurement of distances in this space in two ways. First, we have implemented an Eikonal-type equation using level-set methods to produce a distance transform which respects the metric $G$. This required the derivation of a formula for the speed of an evolving front in the direction of its Euclidean normal. Second, we have implemented Dijkstra's algorithm using $G$ to determine distances between neighboring voxels, using the formula $\left(w^{T} G w\right)^{\frac{1}{2}}$ where $w$ is the vector from a voxel to its neighbor. Though it can suffer from discretization problems, Dijkstra's algorithm is fast and allows interactive display of return paths.

For our level set 9] implementation, we seek a speed function $F$ for use in the evolution equation

$$
\phi_{t}=F|\nabla \phi| .
$$

This can be done using the following algorithm, which amounts to finding the length of the projection of the unit normal in the tensor-warped space onto the Euclidean normal:

1) Set $n=\frac{\nabla \phi}{|\nabla \phi|}$, the Euclidean normal to the level set.

2) Find any two linearly independent vectors $t_{1}$ and $t_{2}$ perpendicular to $n$. These are tangents which span the tangent space to the level set.

3) Set $w=\left(G t_{1}\right) \times\left(G t_{2}\right)$.

4) Set $\tilde{n}=\frac{w}{\left(w^{T} G w\right)^{\frac{1}{2}}}$. This is the unit normal with respect to $G$.

$5)$ Set $F=\left|\tilde{n}^{T} n\right|$. This is the length of the projection of $\tilde{n}$ onto $n$.

\subsection{Experiments}

These experiments were performed on the same data set and with the same preprocessing as in Section 3.1.

Tensor-Warped Distances. Figure 2 shows a slice through a 3D distance transform with respect to the metric derived from the DT-MRI tensor field. The result can be viewed as a topographical map, with iso-level contours. Defining the seed point as the highest elevation, the direction of maximum connectivity will be in the direction of slowest descent.

Connectivity Measure. By comparing the geodesic path length to the Euclidean length of the same path, we produce a measure of the "degree of connectivity" between any two points. We compute the ratio of Euclidean path length to geodesic path length for all paths outward from the initial point. Figure 3 displays the connectivity measure as calculated for the distance map shown in Figure 2, 
Fig. 2. Tensor-warped distance map: this contour map shows metric distance from an initial point located in the posterolateral part of the corpus callosum. The image is a slice through a 3D distance map, at the level of the initial point. The apparent "ridges" in the image indicate low metric distance, or high connectivity.

\section{Discussion}

The introduction of a Riemannian metric allowed us to reformulate the connectivity/diffusion simulation problem as a search for geodesic paths. In addition, we solved for a steady-state heat distribution and flow field which reflect connectivity.

The connection between the anisotropic diffusion operator and the LaplaceBeltrami operator (eq. 12) relates an anisotropic diffusion process in $\mathcal{R}^{3}$ to an isotropic diffusion process in a Riemannian space, where the metric is defined by the inverse of the diffusion tensor. This connection is likely responsible for the observed similarity between the results of both methods. Qualitatively, the steady-state maximal heat flow path between the source and sink appears to follow the geodesic derived from the Riemannian shortest path algorithm. The precise nature of this connection is a topic for further investigation.

\section{Acknowledgements}

This work was funded in part by a National Science Foundation Graduate Fellowship and by NIH grants P41-RR13218 and 1 R33-CA99015. 

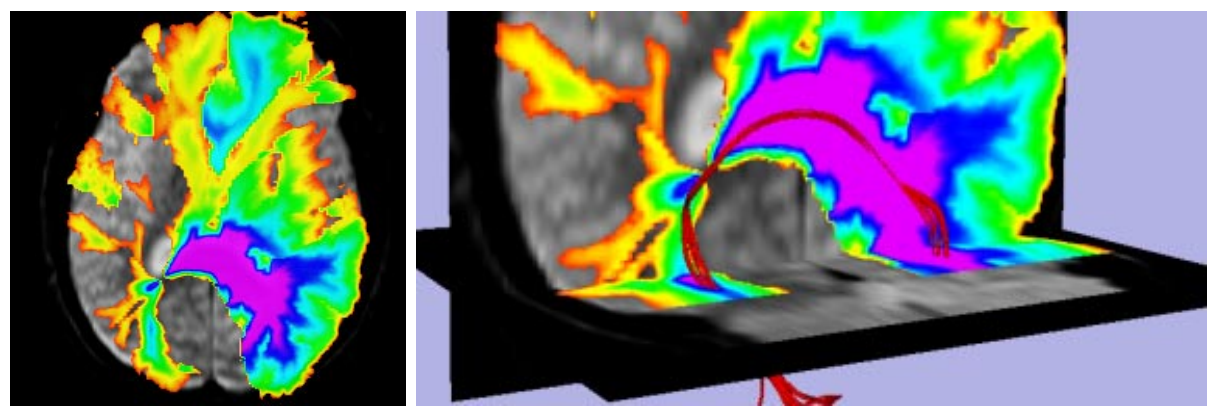

Fig. 3. Degree of connectivity, measured as Euclidean path length over geodesic path length. Very low connectivity is not shown. Purple is the highest connectivity. Traditional tractography based on following the principal eigenvector direction, with seed locations around the initial point, is displayed in red (right). Visual inspection confirms that the trace lines agree well with the region of highest connectivity.

\section{References}

1. P.J. Basser: Inferring microstructural features and the physiological state of tissues from diffusion-weighted images. NMR in Biomedicine. 8 (1995) 333-344.

2. P.J. Basser and S. Pajevic and C. Pierpaoli and J. Duda and A. Aldroubi: In Vivo Fiber Tractography Using DT-MRI Data. Magn. Reson. Med.. 44 (2000) 625-632.

3. P.G. Batchelor, D.L.G. Hill, F. Calamante and D. Atkinson: Study of Connectivity in the Brain Using the Full Diffusion Tensor from MRI. Information Processing in Medical Imaging, 17th International Conference, IPMI'01. (2001) 121-133.

4. M.C. De Lara: Geometric and Symmetry Properties of a Nondegenerate Diffusion Process. Annals of Probability. 23:4 (1995) 1557-1604.

5. G. Kindlmann, D. Weinstein, D. Hart: Strategies for Direct Volume Rendering of Diffusion Tensor Fields. IEEE Transactions on Visualization and Computer Graphics. 6:2 (2000) 124-138.

6. H. Gudbjartsson, S. Maier, R. Mulkern, I.A. Morocz, S. Patz, F. Jolesz: Line scan diffusion imaging. Magn. Reson. Med. (1996) 36 509-519.

7. G.J.M. Parker, C.A.M. Wheeler-Kingshott, and G.J. Barker: Distributed Anatomical Brain Connectivity Derived from Diffusion Tensor Imaging. Information Processing in Medical Imaging 17th International Conference, IPMI'01. (2001) 106120.

8. C. Poupon, C. A. Clark, F. Frouin, J. Régis, I. Bloch, D. Le Bihan, I. Bloch, and J.-F. Mangin: Regularization of Diffusion-Based Direction Maps for the Tracking Brain White Matter Fascicles. NeuroImage. 12 (2000) 184-195.

9. J.A. Sethian: Level Set Methods and Fast Marching Methods. Cambridge University Press. (1999).

10. D.S. Tuch: Diffusion MRI of Complex Tissue Structure. Ph.D. Thesis, Division of Health Sciences and Technology, Massachusetts Institute of Technology. (2002).

11. C.-F. Westin, S. Peled, H. Gudbjartsson, R. Kikinis and F.A. Jolesz. Geometrical Diffusion Measures for MRI from Tensor Basis Analysis. ISMRM 97 Vancouver, Canada (1997).

12. C.-F. Westin, S.E. Maier, H. Mamata, A. Nabavi, F.A. Jolesz, and R. Kikinis.: Processing and Visualization of Diffusion Tensor MRI. Med. Image Anal. 6:2 (2002) 93-108. 